Review Article

\title{
Epidemiological Patterns of Skin Disease in Saudi Arabia: A Systematic Review and Meta-Analysis
}

\author{
Mohammad Almohideb \\ King Saud Bin Abdulaziz University for Health Sciences, College of Medicine, Riyadh, Saudi Arabia \\ Correspondence should be addressed to Mohammad Almohideb; moalm20@gmail.com
}

Received 28 March 2020; Accepted 25 September 2020; Published 28 October 2020

Academic Editor: Markus Stucker

Copyright (C) 2020 Mohammad Almohideb. This is an open access article distributed under the Creative Commons Attribution License, which permits unrestricted use, distribution, and reproduction in any medium, provided the original work is properly cited.

\begin{abstract}
Background. Large epidemiological studies on patterns of skin diseases in Saudi Arabia are scarce. Therefore, this systematic review and meta-analysis was conducted to gather available epidemiologic data describing the pattern of skin diseases in different geographical areas in Saudi Arabia. Methods. A comprehensive literature search of articles was conducted in PubMed, SCOPUS, and Web of Science through October 2019. We included all published cross-sectional studies that provided data on relevant incidence or prevalence of skin disease in Saudi Arabia. The risk of bias within the included cross-sectional studies was assessed using the Hoy tool for the prevalence studies. All statistical analysis was performed using the Comprehensive Meta-analysis software. Results. The present meta-analysis included 14 studies that reported the frequency of the skin disease patterns in different regions in Saudi Arabia with a total sample size of 30436 patients with an overall low risk of bias. The diseases of skin appendages and dermatitis were the most commonly reported skin diseases in Saudi Arabia (24.8\% (95\% CI, 24.3-25.3) and 24\% (95\% CI, $23.6 \%-24.6 \%)$, respectively). Skin infection represented about $18.5 \%$ (95\% CI, 18.1\%-19\%), while the papulosquamous disorders represented 5.3\% (95\% CI, 5\%-5.6\%) of the skin diseases in Saudi Arabia. Skin cancers were pooled from only two studies. Basal cell carcinoma and squamous cell carcinoma were the most common malignant neoplasm in Saudi Arabia (51.4\% and $22.5 \%$ of the malignant neoplasm, respectively), while malignant melanoma represents only $3.8 \%$ of the malignant skin cancer. Conclusion. Adnexal disorders and dermatitis are the most common skin disease in Saudi Arabia, followed by skin infection and pigmentary disorders. While skin cancer is more frequent than other countries, awareness campaigns should be initiated to increase knowledge about the harmful effect of long-term sun exposure.
\end{abstract}

\section{Introduction}

Skin, similar to other human organs, can be affected by all types of pathological changes, including hereditary, inflammatory, neoplastic, endocrinal, traumatic, and degenerative affection [1]. Epidemiological studies are important for understanding the implications of human disease. Identifying the incidence and prevalence of specific diseases is indispensable to decision making regarding the distribution of resources for clinical care and research [2]. For example, the management of skin disorders requires an appropriate diagnosis. Nondermatologists, as in the case of general practitioners, usually perform diagnoses and treatment of skin disorders in underserved areas, which highlights the importance of providing a comprehensive review of the skin disease patterns in each specific region; in addition to further focus on educating nondermatologists regarding some common skin conditions they might encounter [3-5].

The incidence and prevalence of skin disorders are mainly related to the ethnic and the genetic constitution of the community. In addition, hygiene, dietary style, social background, and weather conditions are important contributing factors.

Population-based epidemiological studies of skin disease are relatively inadequate. Because these epidemiologic studies have been published through many decades in different journals, many dermatologists are not aware of these data [2].

In Saudi Arabia, the large epidemiological studies on patterns of skin diseases are scanty. Previous epidemiological 
studies in Saudi Arabia investigated the pattern of skin and skin-related diseases in different regions of Saudi Arabia such as Madinah, Al-Khobar, Jeddah, Al-Baha, Hail, Abha, Qassim, and Najran [6-15], which reflect the pattern of skin and skinrelated diseases in Saudi Arabia.

Therefore, this systematic review and meta-analysis was conducted to gather available epidemiological data describing the pattern of skin diseases and investigate the current evidence of frequency, type, and distribution of skin disorders in different geographical areas in Saudi Arabia.

\section{Methods}

All steps of this systematic review were performed in strict compliance with the Cochrane handbook of systematic reviews and meta-analysis [16] in addition to following the preferred reporting items for systematic reviews and metaanalyses (PRISMA statement guidelines) during the drafting process of this manuscript [17].

2.1. Literature Search Strategy. The following medical electronic databases were searched: PubMed, SCOPUS, and Web of Science through October 2019 using the following query: [(prevalence OR incidence OR epidemiology OR pattern of skin disease OR skin disease pattern)] AND (melanocytic nevi OR Vitiligo OR Melasma OR Dermatitis OR eczema OR acne OR alopecia OR fungal skin disease OR cutaneous leishmaniasis OR scabies OR warts OR chicken pox OR herpes simplex OR herpes zoster OR onychomycosis OR tinea OR dermatophytosis OR candidiasis OR pityriasis versicolorversicolor OR psoriasis OR Lichen planus OR pityriasis rosea OR skin cancer OR skin malignancy OR skin carcinoma OR melanoma) AND Saudi Arabia. The bibliography of eligible studies was searched to find relevant articles.

2.2. Eligibility Criteria and Study Selection. All published cross-sectional studies that provided data on relevant incidence or prevalence of skin disease in Saudi Arabia were included. Excluded articles included all studies with a small sample size (less than 100 patients), reviews, case reports, conference abstracts, or case series, studies with self-reported data unless diagnoses were validated by a trained physician, studies on specific ethnic or social groups, non-English articles, and duplicate references.

Eligibility screening was conducted in two steps, each by two independent reviewers (MA and MA) as follows: (a) title and abstract screening for matching the inclusion criteria and (b) full-text screening for eligibility to meta-analysis. Disagreements were resolved upon the opinion of a third reviewer (HA).

2.3. Data Extraction. Two independent reviewers (MA and MA) extracted the data that included the following: (a) general characteristics of each study including study setting, study design, sample size; (b) patients' baseline characteristics of each study including age, gender, and nationality; (c) types and proportion of the reported skin diseases; (d) risk of bias criteria.

2.4. Risk of Bias Assessment. To assess the risk of bias within the included cross-sectional studies, two independent reviewers (MA and MA) used the risk of bias assessment tool developed by Hoy et al. [18] for the prevalence studies. The domains of risk of bias assessment were presented in Supplementary file 1 .

2.5. Data Synthesis. All statistical analysis was performed using the Comprehensive Meta-Analysis software (CMA version 3) for Windows. The mean proportions of skin diseases were pooled in a meta-analysis model, using the Mantel-Haenszel method. The analysis was performed under the fixed-effects model for homogeneous data and the random-effects model for heterogeneous data. Heterogeneity among studies was assessed using $I^{2}$ test and -value from the chi-squared test of heterogeneity. Values of $I^{2}>50$ are significant determinants of heterogeneity among studies [19].

\section{Results}

3.1. Results of the Literature Search. Our search yielded a total of 1701 studies. Following screening and excluding duplicates, we remained with 95 studies that entered full-text screening. Finally, 14 studies were included in this systematic review as reported in the PRISMA flow diagram (Figure 1).

3.2. Summary of the Included Studies. The fourteen Saudi studies reported the frequency of the skin disease patterns in different regions in Saudi Arabia with a total sample size of 30436 patients. Only two studies reported the pattern of skin cancers only in southeastern and western regions in Saudi Arabia, with a total sample size of 395 patients with skin cancer $[6,10]$. Baseline characteristics and summary of the included studies are reported in Table 1.

Overall low risk of bias was observed in accordance with the Hoy et al. [18] assessment tool for the prevalence studies. Summary of risk of bias assessment is reported in Table 2.

3.3. Pattern of Skin Diseases in Saudi Arabia. The overall mean proportion of pigmentary disorders, as reported by 11 studies (16658 patients), was 16.1\% (95\% CI, 15.4\%-16.9\%) (Figure 2(a). The most common pigmentary disorder was the melanocytic nevi 54.2\% (95\% CI, 52.2\%-56.1\%) followed by postinflammatory pigmentation and vitiligo; $47 \%(95 \%$ CI, 45\%-49\%) and 6\% (95\% CI, 5.6\%-6.3\%), respectively Table 3 and Supplementary file 2. The pooled proportion of melanocytic nevi was significantly higher in males than females: OR 0.48 (95\% CI, 0.33-0.69) (Table 4).

Considering dermatitis or eczema, the pooled proportion form 12 studies (29244 patients) resulted in an overall prevalence of 24\% (95\% CI, 23.6\%-24.6\%) (Figure 2(b)). Contact dermatitis and seborrheic dermatitis were common with a prevalence of $4.7 \%$ (95\% CI $4.3 \%-5.1 \%$ ) and $2.3 \%$ 

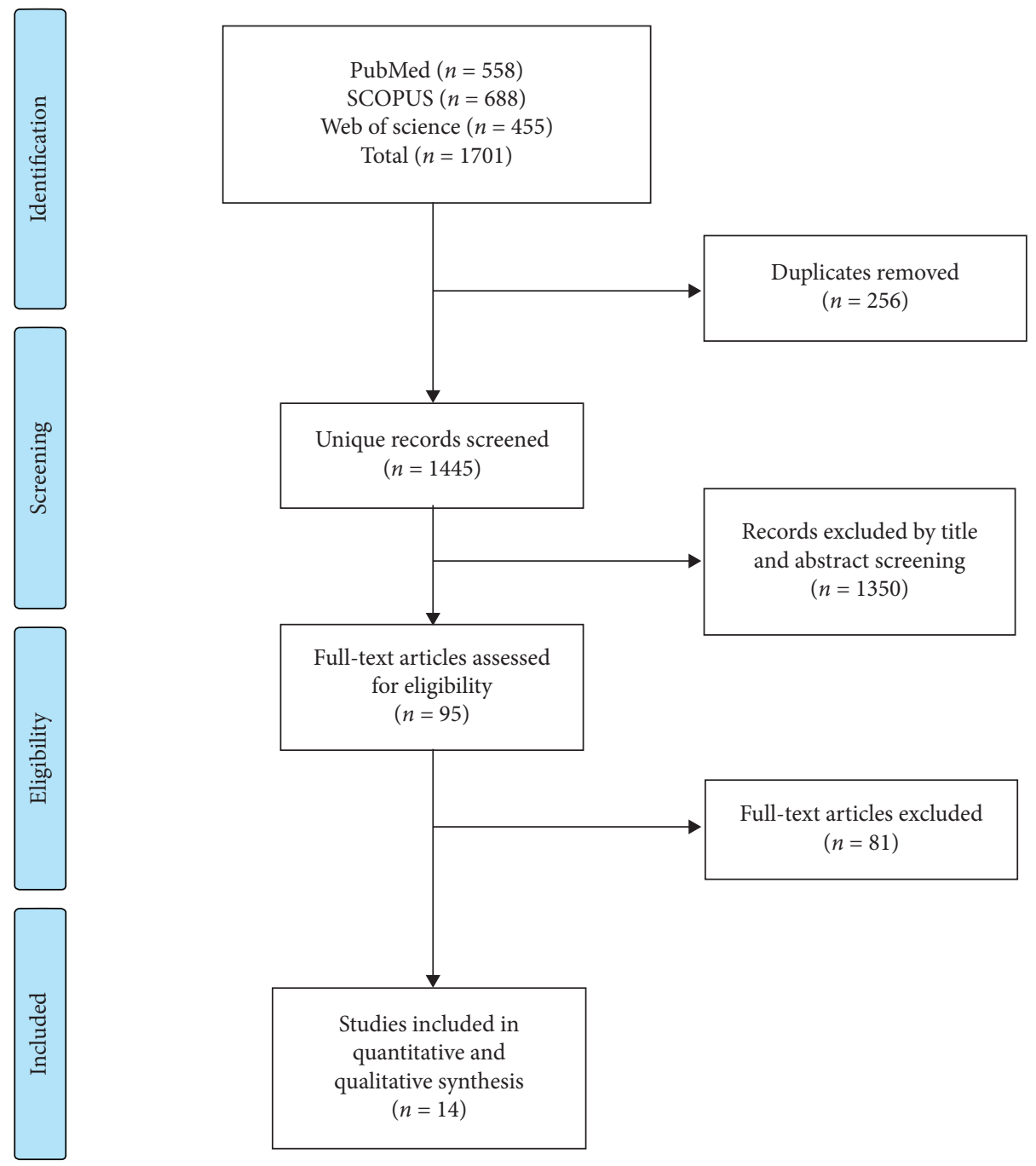

FIgURE 1: PRISMA flow diagram.

(95\% CI, 2\%-2.7\%), respectively (Supplementary file 3). The pooled proportion of contact dermatitis was significantly higher in females than males: OR 0.82 (95\% CI, 0.68-0.98) (Table 4).

Skin infection represented about 18.5\% (95\% CI, 18.1\%$19 \%)$, as reported by 12 studies (29244 patients) (Figure 2(c)). Cutaneous leishmaniasis was the most common parasitic skin diseases $4 \%$ (95\% CI, 3.7\%-4.3\%), while warts were the most common viral infection $7.2 \%(95 \% \mathrm{CI}$, $6.8 \%-7.5 \%$ ) followed by chicken pox $6.9 \%$ (95\% CI, $6.2 \%-$ $7.7 \%$ ). Bacterial skin diseases represented 3.3\% (95\% CI, 3\%$3.6 \%)$. The most commonly reported fungal infections were dermatophytosis $6.6 \%(95 \% \mathrm{CI}, 5.8 \%-7.4 \%)$ and onychomycosis $2.8 \%$ (95\% CI, 2.3\%-3.4\%) (Supplementary file 4).

The pooled proportion of cutaneous leishmaniasis and warts was significantly higher in females than males: OR 2.07 (95\% CI, 1.58-2.72) and OR 1.49 (95\%CI, 1.25-1.77), while the bacterial infection was more common in males: OR 1.80 (95\% CI, 1.41-2.30) (Table 4).

Regarding the diseases of skin appendages, the mean proportion was $24.8 \%$ (95\% CI, 24.3-25.3) (Figure 2(d)) as reported by 9 studies (27177 patients). Alopecia and acne served a proportion of 7.5\% (95\% CI, 7.1\%-7.8\%) and $1.8 \%$ (95\% CI, 1.7\%-1.8\%) (Supplementary file 5). The pooled proportion of acne was significantly higher in females than males: OR 0.57 (95\% CI, 0.49-0.65) (Table 4).

On the other hand, papulosquamous disorders represented $5.3 \%(95 \% \mathrm{CI}, 5 \%-5.6 \%)$ of the skin diseases in Saudi Arabia (Figure 2(e)), as reported by 11 studies (30076 patients). Psoriasis represents 3.9\% (95\% CI, $3.6 \%-4.1 \%$ ), while Lichen planus represents $1.8 \%$ (95\% CI, $1 \%-1.5 \%$ ) (Supplementary file 6 ). The summary of the pooled proportion of the skin diseases pattern in Saudi Arabia is presented in Table 2.

Finally, the overall mean proportion of benign skin neoplasms, as reported by two studies (1244 patients) was 21.2\% (95\% CI, 18\%-24.7\%) (Figure 3(a)), while malignant neoplasms were $5 \%$. Two studies reported the histological classification of skin cancer in Saudi Arabia. The pooled proportion from the two studies is reported in Figure 4. Basal cell carcinoma and squamous cell carcinoma were the most common malignant neoplasm in Saudi Arabia (51.4\% 


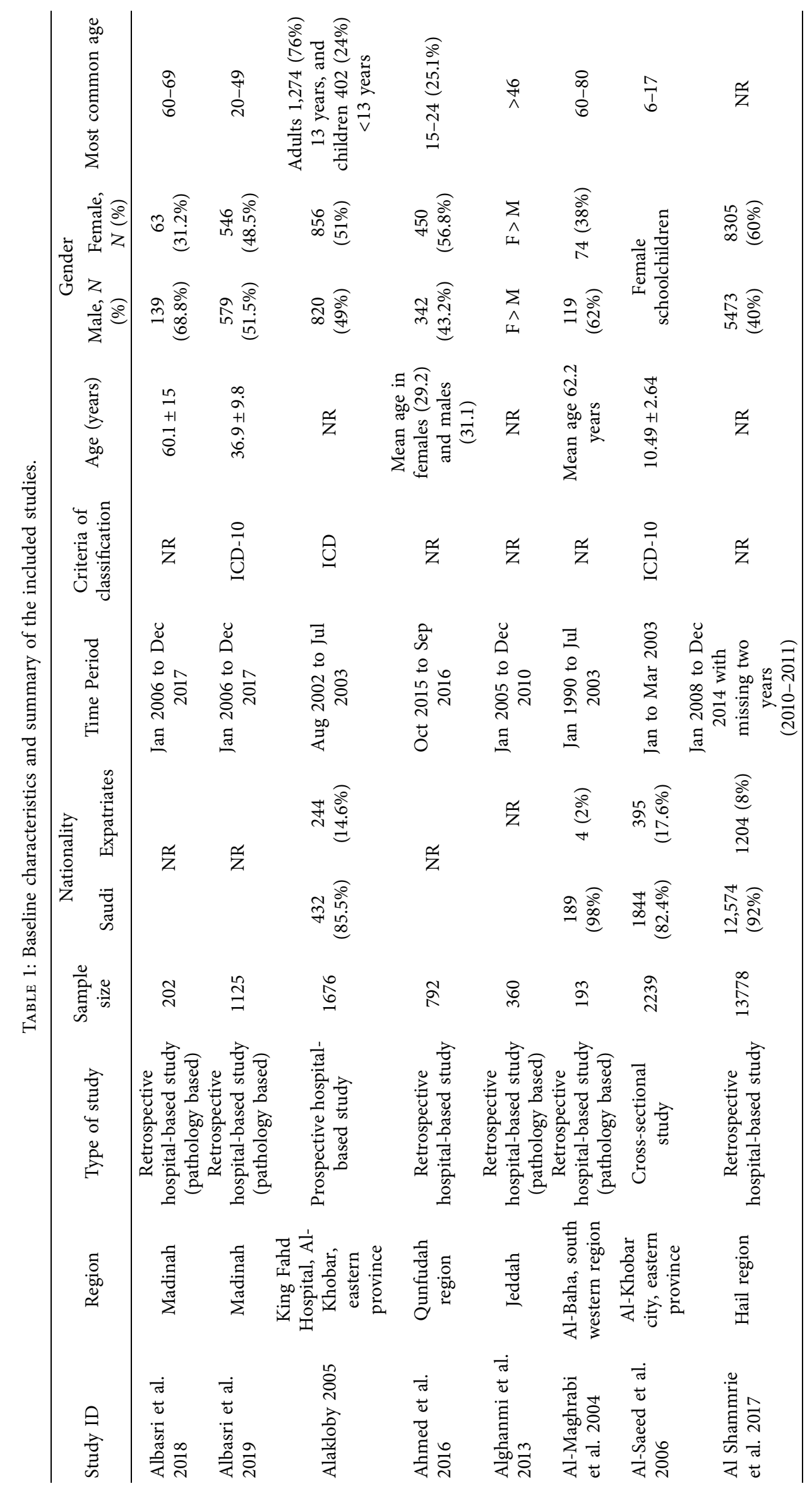




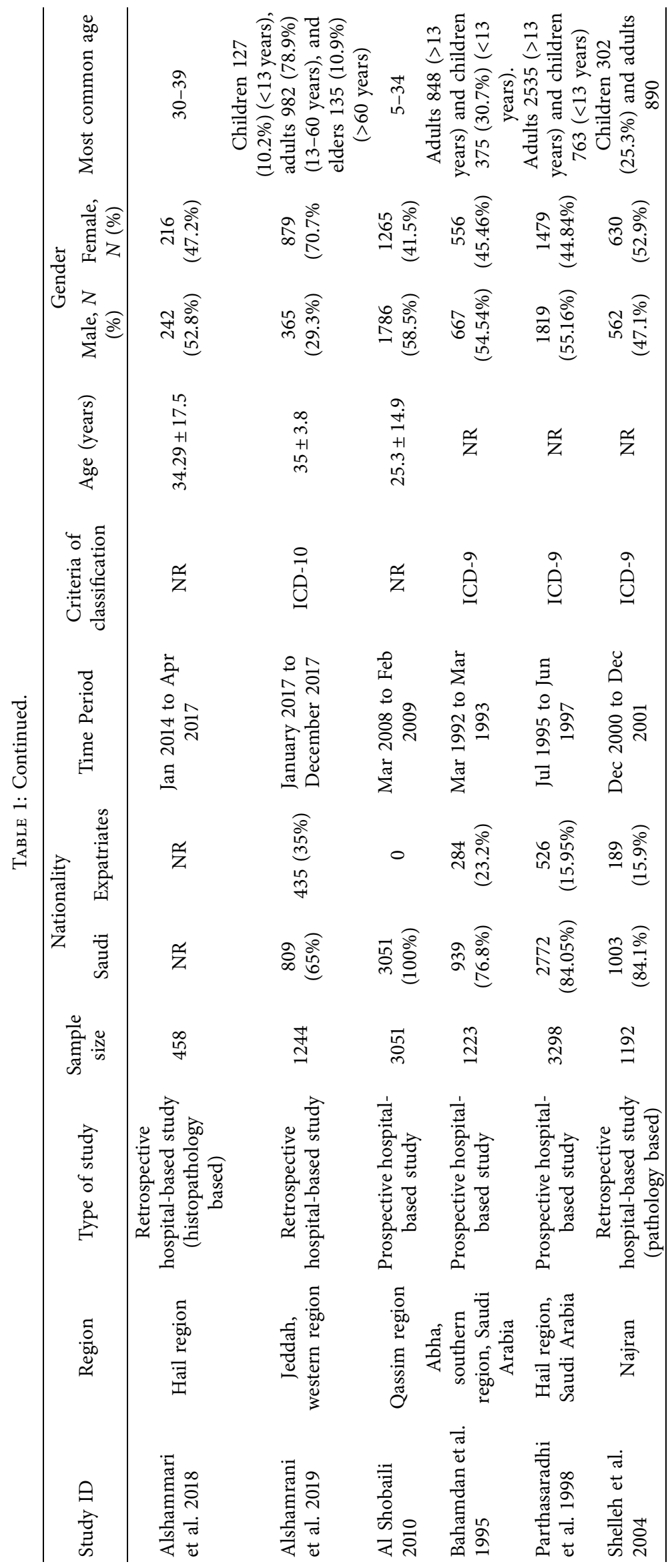


TABLE 2: Evaluation of the risk of bias in included primary studies.

\begin{tabular}{|c|c|c|c|c|c|c|c|c|c|c|c|}
\hline Study ID & Q1 & Q2 & Q3 & Q4 & Q5 & Q6 & Q7 & Q8 & Q9 & Q10 & $\begin{array}{l}\text { Overall risk of } \\
\text { study bias }\end{array}$ \\
\hline Albasri et al. 2018 & $\begin{array}{l}\text { Low } \\
\text { risk }\end{array}$ & $\begin{array}{l}\text { Low } \\
\text { risk }\end{array}$ & $\begin{array}{l}\text { Low } \\
\text { risk }\end{array}$ & $\begin{array}{l}\text { Low } \\
\text { risk }\end{array}$ & $\begin{array}{l}\text { Low } \\
\text { risk }\end{array}$ & $\begin{array}{l}\text { Low } \\
\text { risk }\end{array}$ & $\begin{array}{l}\text { Low } \\
\text { risk }\end{array}$ & $\begin{array}{l}\text { Low } \\
\text { risk }\end{array}$ & $\begin{array}{l}\text { Low } \\
\text { risk }\end{array}$ & $\begin{array}{l}\text { Low } \\
\text { risk }\end{array}$ & Low risk \\
\hline Albasri et al. 2019 & $\begin{array}{l}\text { High } \\
\text { risk }\end{array}$ & $\begin{array}{l}\text { Low } \\
\text { risk }\end{array}$ & $\begin{array}{l}\text { Low } \\
\text { risk }\end{array}$ & $\begin{array}{l}\text { Low } \\
\text { risk }\end{array}$ & $\begin{array}{l}\text { Low } \\
\text { risk }\end{array}$ & $\begin{array}{l}\text { Low } \\
\text { risk }\end{array}$ & $\begin{array}{l}\text { Low } \\
\text { risk }\end{array}$ & $\begin{array}{l}\text { Low } \\
\text { risk }\end{array}$ & $\begin{array}{l}\text { Low } \\
\text { risk }\end{array}$ & $\begin{array}{l}\text { Low } \\
\text { risk }\end{array}$ & Low risk \\
\hline Alakloby 2005 & $\begin{array}{l}\text { High } \\
\text { risk }\end{array}$ & $\begin{array}{l}\text { Low } \\
\text { risk }\end{array}$ & $\begin{array}{l}\text { Low } \\
\text { risk }\end{array}$ & $\begin{array}{l}\text { Low } \\
\text { risk }\end{array}$ & $\begin{array}{l}\text { Low } \\
\text { risk }\end{array}$ & $\begin{array}{l}\text { Low } \\
\text { risk }\end{array}$ & $\begin{array}{l}\text { Low } \\
\text { risk }\end{array}$ & $\begin{array}{l}\text { Low } \\
\text { risk }\end{array}$ & $\begin{array}{l}\text { Low } \\
\text { risk }\end{array}$ & $\begin{array}{l}\text { Low } \\
\text { risk }\end{array}$ & Low risk \\
\hline Ahmed et al. 2016 & $\begin{array}{l}\text { High } \\
\text { risk }\end{array}$ & $\begin{array}{l}\text { High } \\
\text { risk }\end{array}$ & $\begin{array}{l}\text { High } \\
\text { risk }\end{array}$ & $\begin{array}{l}\text { Low } \\
\text { risk }\end{array}$ & $\begin{array}{l}\text { Low } \\
\text { risk }\end{array}$ & $\begin{array}{l}\text { Low } \\
\text { risk }\end{array}$ & $\begin{array}{l}\text { High } \\
\text { risk }\end{array}$ & $\begin{array}{l}\text { Low } \\
\text { risk }\end{array}$ & $\begin{array}{l}\text { Low } \\
\text { risk }\end{array}$ & $\begin{array}{l}\text { Low } \\
\text { risk }\end{array}$ & Moderate risk \\
\hline Alghanmi et al. 2013 & $\begin{array}{l}\text { Low } \\
\text { risk }\end{array}$ & $\begin{array}{l}\text { Low } \\
\text { risk }\end{array}$ & $\begin{array}{l}\text { Low } \\
\text { risk }\end{array}$ & $\begin{array}{l}\text { Low } \\
\text { risk }\end{array}$ & $\begin{array}{l}\text { Low } \\
\text { risk }\end{array}$ & $\begin{array}{l}\text { Low } \\
\text { risk }\end{array}$ & $\begin{array}{l}\text { Low } \\
\text { risk }\end{array}$ & $\begin{array}{l}\text { Low } \\
\text { risk }\end{array}$ & $\begin{array}{l}\text { Low } \\
\text { risk }\end{array}$ & $\begin{array}{l}\text { Low } \\
\text { risk }\end{array}$ & Low risk \\
\hline $\begin{array}{l}\text { Al-Maghrabi et al. } \\
2004\end{array}$ & $\begin{array}{l}\text { Low } \\
\text { risk }\end{array}$ & $\begin{array}{l}\text { Low } \\
\text { risk }\end{array}$ & $\begin{array}{l}\text { Low } \\
\text { risk }\end{array}$ & $\begin{array}{l}\text { Low } \\
\text { risk }\end{array}$ & $\begin{array}{l}\text { Low } \\
\text { risk }\end{array}$ & $\begin{array}{l}\text { Low } \\
\text { risk }\end{array}$ & $\begin{array}{l}\text { Low } \\
\text { risk }\end{array}$ & $\begin{array}{l}\text { Low } \\
\text { risk }\end{array}$ & $\begin{array}{l}\text { Low } \\
\text { risk }\end{array}$ & $\begin{array}{l}\text { Low } \\
\text { risk }\end{array}$ & Low risk \\
\hline Al-Saeed et al. 2006 & $\begin{array}{l}\text { High } \\
\text { risk }\end{array}$ & $\begin{array}{l}\text { Low } \\
\text { risk }\end{array}$ & $\begin{array}{l}\text { Low } \\
\text { risk }\end{array}$ & $\begin{array}{l}\text { Low } \\
\text { risk }\end{array}$ & $\begin{array}{l}\text { Low } \\
\text { risk }\end{array}$ & $\begin{array}{l}\text { Low } \\
\text { risk }\end{array}$ & $\begin{array}{l}\text { Low } \\
\text { risk }\end{array}$ & $\begin{array}{l}\text { Low } \\
\text { risk }\end{array}$ & $\begin{array}{l}\text { High } \\
\text { risk }\end{array}$ & $\begin{array}{l}\text { Low } \\
\text { risk }\end{array}$ & Low risk \\
\hline $\begin{array}{l}\text { Al Shammrie et al. } \\
2017\end{array}$ & $\begin{array}{l}\text { Low } \\
\text { risk }\end{array}$ & $\begin{array}{l}\text { Low } \\
\text { risk }\end{array}$ & $\begin{array}{l}\text { Low } \\
\text { risk }\end{array}$ & $\begin{array}{l}\text { Low } \\
\text { risk }\end{array}$ & $\begin{array}{l}\text { Low } \\
\text { risk }\end{array}$ & $\begin{array}{l}\text { Low } \\
\text { risk }\end{array}$ & $\begin{array}{l}\text { Low } \\
\text { risk }\end{array}$ & $\begin{array}{l}\text { Low } \\
\text { risk }\end{array}$ & $\begin{array}{l}\text { High } \\
\text { risk }\end{array}$ & $\begin{array}{l}\text { Low } \\
\text { risk }\end{array}$ & Low risk \\
\hline $\begin{array}{l}\text { Alshammari et al. } \\
2018\end{array}$ & $\begin{array}{l}\text { High } \\
\text { risk }\end{array}$ & $\begin{array}{l}\text { Low } \\
\text { risk }\end{array}$ & $\begin{array}{l}\text { Low } \\
\text { risk }\end{array}$ & $\begin{array}{l}\text { Low } \\
\text { risk }\end{array}$ & $\begin{array}{l}\text { Low } \\
\text { risk }\end{array}$ & $\begin{array}{l}\text { Low } \\
\text { risk }\end{array}$ & $\begin{array}{l}\text { Low } \\
\text { risk }\end{array}$ & $\begin{array}{l}\text { Low } \\
\text { risk }\end{array}$ & $\begin{array}{l}\text { Low } \\
\text { risk }\end{array}$ & $\begin{array}{l}\text { Low } \\
\text { risk }\end{array}$ & Low risk \\
\hline $\begin{array}{l}\text { Alshamrani et al. } \\
2019\end{array}$ & $\begin{array}{l}\text { High } \\
\text { risk }\end{array}$ & $\begin{array}{l}\text { Low } \\
\text { risk }\end{array}$ & $\begin{array}{l}\text { Low } \\
\text { risk }\end{array}$ & $\begin{array}{l}\text { Low } \\
\text { risk }\end{array}$ & $\begin{array}{l}\text { High } \\
\text { risk }\end{array}$ & $\begin{array}{l}\text { Low } \\
\text { risk }\end{array}$ & $\begin{array}{l}\text { Low } \\
\text { risk }\end{array}$ & $\begin{array}{l}\text { Low } \\
\text { risk }\end{array}$ & $\begin{array}{l}\text { High } \\
\text { risk }\end{array}$ & $\begin{array}{l}\text { Low } \\
\text { risk }\end{array}$ & Low risk \\
\hline Al Shobaili 2010 & $\begin{array}{l}\text { Low } \\
\text { risk }\end{array}$ & $\begin{array}{l}\text { Low } \\
\text { risk }\end{array}$ & $\begin{array}{l}\text { Low } \\
\text { risk }\end{array}$ & $\begin{array}{l}\text { Low } \\
\text { risk }\end{array}$ & $\begin{array}{l}\text { Low } \\
\text { risk }\end{array}$ & $\begin{array}{l}\text { Low } \\
\text { risk }\end{array}$ & $\begin{array}{l}\text { Low } \\
\text { risk }\end{array}$ & $\begin{array}{l}\text { Low } \\
\text { risk }\end{array}$ & $\begin{array}{l}\text { Low } \\
\text { risk }\end{array}$ & $\begin{array}{l}\text { Low } \\
\text { risk }\end{array}$ & Low risk \\
\hline Bahamdan et al. 1995 & $\begin{array}{l}\text { Low } \\
\text { risk }\end{array}$ & $\begin{array}{l}\text { Low } \\
\text { risk }\end{array}$ & $\begin{array}{l}\text { Low } \\
\text { risk }\end{array}$ & $\begin{array}{l}\text { Low } \\
\text { risk }\end{array}$ & $\begin{array}{l}\text { Low } \\
\text { risk }\end{array}$ & $\begin{array}{l}\text { Low } \\
\text { risk }\end{array}$ & $\begin{array}{l}\text { Low } \\
\text { risk }\end{array}$ & $\begin{array}{l}\text { Low } \\
\text { risk }\end{array}$ & $\begin{array}{l}\text { Low } \\
\text { risk }\end{array}$ & $\begin{array}{l}\text { Low } \\
\text { risk }\end{array}$ & Low risk \\
\hline $\begin{array}{l}\text { Parthasaradhi et al. } \\
1998\end{array}$ & $\begin{array}{l}\text { Low } \\
\text { risk }\end{array}$ & $\begin{array}{l}\text { Low } \\
\text { risk }\end{array}$ & $\begin{array}{l}\text { Low } \\
\text { risk }\end{array}$ & $\begin{array}{l}\text { Low } \\
\text { risk }\end{array}$ & $\begin{array}{l}\text { Low } \\
\text { risk }\end{array}$ & $\begin{array}{l}\text { Low } \\
\text { risk }\end{array}$ & $\begin{array}{l}\text { Low } \\
\text { risk }\end{array}$ & $\begin{array}{l}\text { Low } \\
\text { risk }\end{array}$ & $\begin{array}{l}\text { Low } \\
\text { risk }\end{array}$ & $\begin{array}{l}\text { Low } \\
\text { risk }\end{array}$ & Low risk \\
\hline Shelleh et al. 2004 & $\begin{array}{l}\text { Low } \\
\text { risk }\end{array}$ & $\begin{array}{l}\text { Low } \\
\text { risk }\end{array}$ & $\begin{array}{l}\text { Low } \\
\text { risk }\end{array}$ & $\begin{array}{l}\text { Low } \\
\text { risk }\end{array}$ & $\begin{array}{l}\text { Low } \\
\text { risk }\end{array}$ & $\begin{array}{l}\text { Low } \\
\text { risk }\end{array}$ & $\begin{array}{l}\text { Low } \\
\text { risk }\end{array}$ & $\begin{array}{l}\text { Low } \\
\text { risk }\end{array}$ & $\begin{array}{l}\text { Low } \\
\text { risk }\end{array}$ & $\begin{array}{l}\text { Low } \\
\text { risk }\end{array}$ & Low risk \\
\hline
\end{tabular}

List of the 10 questions (Q1-Q10) applied to the studies: Q1. Was the study's target population a close representation of the national population in relation to relevant variables, e.g., age, sex, occupation? Q2. Was the sampling frame a true or close representation of the target population? Q3. Was some form of random selection used to select the sample, OR, was a census undertaken? Q4. Was the likelihood of nonresponse bias minimal? Q5. Were data collected directly from the subjects (as opposed to a proxy)? Q6. Was an acceptable case definition used in the study? Q7. Was the study instrument that measured the parameter of interest shown to have reliability and validity (if necessary)? Q8. Was the same mode of data collection used for all subjects? Q9. Was the length of the shortest prevalence period for the parameter of interest appropriate? Q10. Were the numerator(s) and denominator(s) for the parameter of interest appropriate?

and $22.5 \%$, respectively). Malignant melanoma represents $3.8 \%$ of malignant skin cancers in Saudi Arabia.

\section{Discussion}

This systematic review presented a summary of populationbased data describing the pattern of skin disease in Saudi Arabia. Previous studies investigated the pattern of skin and skin-related diseases in different regions of Saudi Arabia such as Madinah, Al-Khobar, Jeddah, Al-Baha, Hail, Abha, Qassim, and Najran through population-based cross-sectional studies, which reflect the true pattern of skin diseases in Saudi Arabia [6-15]. Although these included data did not cover every part of Saudi Arabia, the results are generalizable to other populations and reflect the current evidence of the frequency, type, and distribution of skin diseases in different geographical areas in Saudi Arabia.

The different patterns of skin disorders in different cities in Saudi Arabia might be justified by overcrowding and/or poor living conditions in some cities. Other ecological and environmental considerations should be considered.
Fourteen Saudi studies with a total sample size of 30436 were included in this systematic review and meta-analysis reporting the frequency of the skin disease patterns in different regions in Saudi Arabia. The average sample size of the individual studies is 2000 patients, which is small to determine the true pattern of skin disease in a large country as Saudi Arabia.

In the present study, dermatitis or eczema represented a high prevalence of skin disorders in Saudi Arabia, with an overall prevalence of $24 \%$ from a total sample size of 29244 patients. Contact dermatitis and seborrheic dermatitis were common. This prevalence is supported by various studies conducted in Saudi Arabia with reported prevalences of $19.6 \%$ [8], 48.2\% [20], 37\% [12], 21\% [21], 19.5\% [14], $25.7 \%$ [15], $16.31 \%$ [22], and $37 \%$ [23]. The high prevalence of dermatitis may contribute to climatical variability in different parts of Saudi Arabia.

Skin infections and infestations prevalence have been reported with an overall pooled proportion of $18.5 \%$ derived from 12 studies with an overall sample size of 29244 patients.

Cutaneous leishmaniasis was the most common parasitic skin diseases, while the warts were the most common viral 
Pigmentary disorder

\begin{tabular}{|c|c|c|c|c|c|c|c|c|c|}
\hline \multirow[t]{2}{*}{ Study name } & \multicolumn{5}{|c|}{ Statistics for each study } & & \multicolumn{3}{|c|}{ Event rate and $95 \% \mathrm{CI}$} \\
\hline & $\begin{array}{c}\text { Event } \\
\text { rate }\end{array}$ & $\begin{array}{c}\text { Lower } \\
\text { limit }\end{array}$ & $\begin{array}{c}\text { Upper } \\
\text { limit }\end{array}$ & $Z$-Value & $p$-Value & & & & \\
\hline A M. Albasri et al., 2019 & 0.119 & 0.101 & 0.139 & -21.739 & 0.000 & & & | & \\
\hline AlaKloby, 2005 & 0.097 & 0.083 & 0.112 & -27.036 & 0.000 & & & 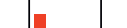 & \\
\hline Ahmed et al., 2016 & 0.077 & 0.060 & 0.098 & -18.635 & 0.000 & & & - & \\
\hline Alghanmi et al., 2013 & 0.014 & 0.006 & 0.033 & -9.465 & 0.000 & & & . & \\
\hline Al-Saeed et al., 2006 & 0.916 & 0.904 & 0.927 & -31.360 & 0.000 & & & & a \\
\hline Alshammari et al., 2018 & 0.212 & 0.177 & 0.252 & -11.491 & 0.000 & & & - & \\
\hline Alshamrani et al., 2019 & 0.044 & 0.034 & 0.057 & -22.284 & 0.000 & & & 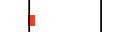 & \\
\hline Al Shobaili, 2010 & 0.112 & 0.101 & 0.123 & -36.075 & 0.000 & & & 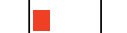 & \\
\hline Bahamdan et al., 1995 & 0.048 & 0.038 & 0.062 & -22.346 & 0.000 & & & 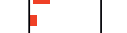 & \\
\hline Parthasaradhi et al., 1998 & 0.071 & 0.062 & 0.080 & -37.918 & 0.000 & & & 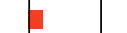 & \\
\hline \multirow[t]{3}{*}{ Shelleh et al., 2004} & 0.108 & 0.092 & 0.127 & -22.621 & 0.000 & & & E & \\
\hline & 0.161 & 0.154 & 0.169 & -59.844 & 0.000 & & & 1 & \\
\hline & & & & & & -1.00 & -0.50 & 0.00 & 1.00 \\
\hline
\end{tabular}

(a)

Dermatitis/eczema and related conditions

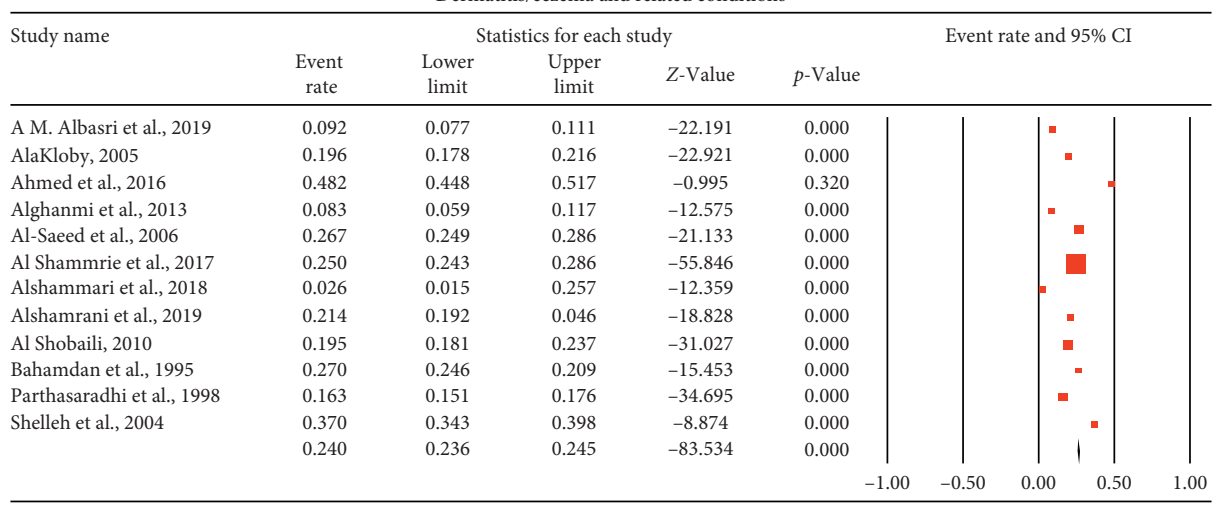

(b)

Infectious diseases

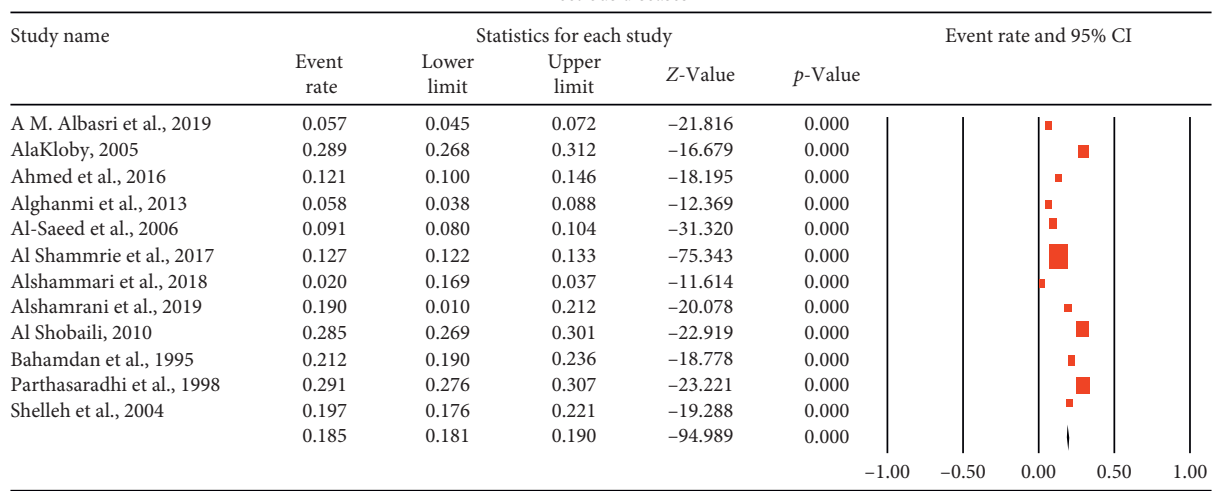

(c)

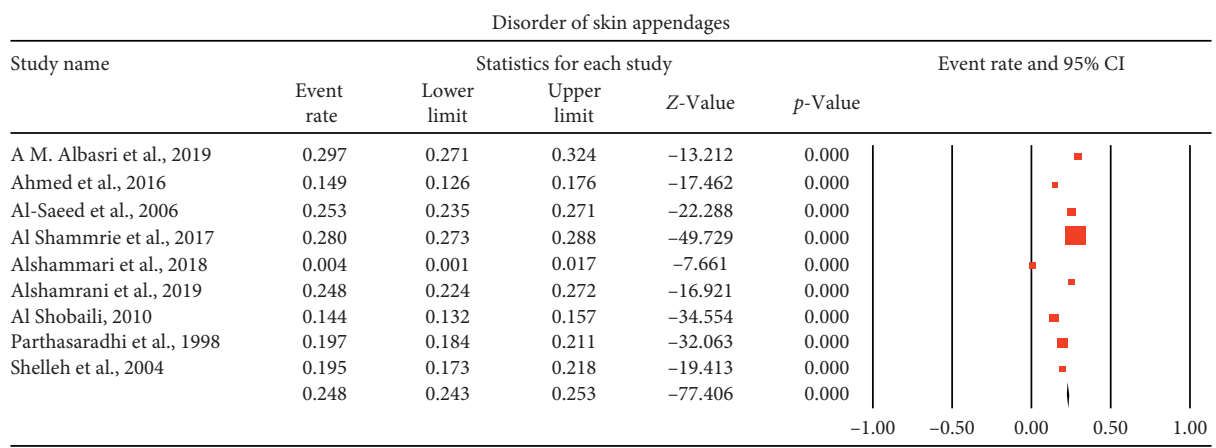

(d)

FIgURE 2: Continued. 


\begin{tabular}{|c|c|c|c|c|c|c|c|c|c|}
\hline \multicolumn{10}{|c|}{ Papulosquamous disorders } \\
\hline \multirow[t]{2}{*}{ Study name } & \multicolumn{5}{|c|}{ Statistics for each study } & \multicolumn{4}{|c|}{ Event rate and $95 \% \mathrm{CI}$} \\
\hline & $\begin{array}{c}\text { Event } \\
\text { rate }\end{array}$ & $\begin{array}{c}\text { Lower } \\
\text { limit }\end{array}$ & $\begin{array}{l}\text { Upper } \\
\text { limit }\end{array}$ & $Z$-Value & $p$-Value & & & & \\
\hline A M. Albasri et al., 2019 & 0.114 & 0.096 & 0.134 & -21.863 & 0.000 & & $1=$ & & \\
\hline AlaKloby, 2005 & 0.064 & 0.053 & 0.077 & -26.876 & 0.000 & & - & & \\
\hline Ahmed et al., 2016 & 0.042 & 0.030 & 0.058 & -17.633 & 0.000 & &. & & \\
\hline Al-Saeed et al., 2006 & 0.004 & 0.002 & 0.008 & -16.504 & 0.000 & & | & & \\
\hline Al Shammrie et al., 2017 & 0.040 & 0.037 & 0.043 & -73.118 & 0.000 & & 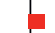 & & \\
\hline Alshammari et al., 2018 & 0.026 & 0.015 & 0.046 & -12.359 & 0.000 & & 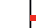 & & \\
\hline Alshamrani et al., 2019 & 0.080 & 0.066 & 0.096 & -23.368 & 0.000 & & - & & \\
\hline Al Shobaili, 2010 & 0.052 & 0.044 & 0.060 & -35.587 & 0.000 & & $=$ & & \\
\hline Bahamdan et al., 1995 & 0.051 & 0.040 & 0.064 & -22.478 & 0.000 & & " & & \\
\hline Parthasaradhi et al., 1998 & 0.062 & 0.054 & 0.071 & -37.616 & 0.000 & & $\square$ & & \\
\hline \multirow[t]{3}{*}{ Shelleh et al., 2004} & 0.067 & 0.054 & 0.083 & -22.737 & 0.000 & & $=$ & & \\
\hline & 0.053 & 0.050 & 0.056 & -106.335 & 0.000 & & | & & \\
\hline & \multicolumn{5}{|r|}{-1.00} & -0.50 & 0.00 & 0.50 & 1.00 \\
\hline
\end{tabular}

(e)

FIGURE 2: Forest plot of the pooled proportion for the prevalence of (a) pigmentary disorders, (b) dermatitis, (c) infectious diseases, (d) disorders of skin appendages, and (e) papulosquamous disorders.

TABle 3: Pooled proportion of the skin diseases pattern in Saudi Arabia.

\begin{tabular}{|c|c|c|}
\hline Skin diseases & Pooled proportion (\%) & $95 \% \mathrm{CI}$ \\
\hline \multicolumn{3}{|l|}{ Pigmentary Disorder } \\
\hline Melanocytic nevi & 54.2 & $52.2 \%-56.1 \%$ \\
\hline Postinflammatory hypo- and hyperpigmentation & 47 & $45 \%-49 \%$ \\
\hline Vitiligo & 6 & $5.6 \%-6.3 \%$ \\
\hline Melasma & 2.5 & $2.2 \%-3 \%$ \\
\hline \multicolumn{3}{|l|}{ Dermatitis/eczema and related conditions } \\
\hline Contact dermatitis & 4.7 & $4.3 \%-5.1 \%$ \\
\hline Seborrheic dermatitis & 2.3 & $2 \%-2.7 \%$ \\
\hline Atopic dermatitis & 1.2 & $1.1 \%-1.3 \%$ \\
\hline Pityriasis alba & 1.6 & $1.2 \%-2.1 \%$ \\
\hline \multicolumn{3}{|l|}{ Disorder of skin appendages } \\
\hline Acne & 1.8 & $1.7 \%-1.8 \%$ \\
\hline Alopecia & 7.5 & $7.1 \%-7.8 \%$ \\
\hline \multicolumn{3}{|l|}{ Infectious diseases } \\
\hline Cutaneous leishmaniasis & 4 & $3.7 \%-4.3 \%$ \\
\hline Scabies & 0.6 & $0.4 \%-0.8 \%$ \\
\hline Warts & 7.2 & $6.8 \%-7.5 \%$ \\
\hline Chicken pox & 6.9 & $6.2 \%-7.7 \%$ \\
\hline Herpes simplex & 1.2 & $0.9 \%-1.5 \%$ \\
\hline Herpes zoster & 1.8 & $1.5 \%-2.2 \%$ \\
\hline Bacterial & 3.3 & $3 \%-3.6 \%$ \\
\hline Onychomycosis & 2.8 & $2.3 \%-3.4 \%$ \\
\hline Tinea & 2.3 & $2.1 \%-2.5 \%$ \\
\hline Dermatophytosis & 6.6 & $5.8 \%-7.4 \%$ \\
\hline Candidiasis & 1.6 & $1.3 \%-2 \%$ \\
\hline Pityriasis versicolor & 1.2 & $0.8 \%-1.7 \%$ \\
\hline \multicolumn{3}{|l|}{ Papulosquamous disorders } \\
\hline Psoriasis & 3.9 & $3.6 \%-4.1 \%$ \\
\hline Lichen planus & 1.8 & $1.6 \%-2.1 \%$ \\
\hline Pityriasis rosea & 1.2 & $1 \%-1.5 \%$ \\
\hline
\end{tabular}

infection followed by chickenpox. In addition, bacterial skin diseases represented $3.3 \%$ of the total prevalence of skin disease in Saudi Arabia.

Similar to the results of this study, a review of populationbased studies from the Rochester epidemiology project showed that skin-related infections and infestations have a high prevalence rate. The incidence of herpes zoster has repeatedly been found to be very high (590 Per 100,000 person-years). The incidence of lower extremity cellulitis is also very common (213 Per 100,000 person-years) [2].

A recent study reported that $10.9 \%$ of the population had a fungal infection in Jeddah, Saudi Arabia [21], which is almost equal to that in Al-Khobar (9.6\%) [21]. This high prevalence of fungal infection is probably attributed to the 
TABLE 4: Comparison between males and females regarding the skin diseases pattern in Saudi Arabia.

\begin{tabular}{|c|c|c|c|c|c|}
\hline Skin diseases & Males & Total & Females & Total & Odds ratio $(95 \% \mathrm{CI})$ \\
\hline \multicolumn{6}{|l|}{ Pigmentary Disorder } \\
\hline Melanocytic nevi & 48 & 944 & 92 & 1425 & $0.48(0.33,0.69)$ \\
\hline Postinflammatory hypo and hyperpigmentation & 20 & 1185 & 41 & 1735 & $0.58(0.33,1.00)$ \\
\hline Vitiligo & 149 & 3671 & 119 & 3770 & $1.20(0.94,1.55)$ \\
\hline Melasma & 18 & 3218 & 86 & 2881 & $0.18(0.11,0.31)$ \\
\hline \multicolumn{6}{|l|}{ Dermatitis/eczema and related conditions } \\
\hline Contact dermatitis & 222 & 4812 & 270 & 4946 & $0.82(0.68,0.98)$ \\
\hline Seborrheic dermatitis & 93 & 4250 & 88 & 4316 & $1.13(0.84,1.52)$ \\
\hline Atopic dermatitis & 501 & 4812 & 487 & 4946 & $1.11(0.97,1.27)$ \\
\hline \multicolumn{6}{|l|}{ Disorder of skin appendages } \\
\hline Acne & 353 & 3566 & 636 & 3844 & $0.57(0.49,0.65)$ \\
\hline Alopecia & 179 & 4145 & 378 & 4390 & $0.48(0.40,0.58)$ \\
\hline \multicolumn{6}{|l|}{ Infectious diseases } \\
\hline Cutaneous leishmaniasis & 185 & 4250 & 78 & 4316 & $2.07(1.58,2.72)$ \\
\hline Scabies & 25 & 3671 & 21 & 3770 & $1.19(0.67,2.12)$ \\
\hline Warts & 356 & 4250 & 239 & 4316 & $1.49(1.25,1.77)$ \\
\hline Chicken pox & 46 & 3306 & 25 & 2891 & $1.53(0.94,2.50)$ \\
\hline Herpes simplex & 28 & 3671 & 21 & 3770 & $1.44(0.80,2.58)$ \\
\hline Herpes zoster & 72 & 3430 & 62 & 3460 & $1.23(0.86,1.75)$ \\
\hline Bacterial & 190 & 4549 & 118 & 4169 & $1.80(1.41,2.30)$ \\
\hline Dermatophytosis & 192 & 3430 & 157 & 3460 & $1.53(1.22,1.91)$ \\
\hline Candidiasis & 41 & 3671 & 53 & 3770 & $0.69(0.46,1.05)$ \\
\hline Pityriasis versicolor & 49 & 1852 & 28 & 2291 & $2.22(1.37,3.58)$ \\
\hline \multicolumn{6}{|l|}{ Papulosquamous disorders } \\
\hline Psoriasis & 163 & 4250 & 159 & 4316 & $1.11(0.88,1.39)$ \\
\hline Lichen planus & 63 & 4250 & 84 & 4316 & $0.78(0.56,1.09)$ \\
\hline Pityriasis rosea & 46 & 4250 & 55 & 4316 & $0.83(0.56,1.22)$ \\
\hline
\end{tabular}

\begin{tabular}{|c|c|c|c|c|c|c|c|c|}
\hline \multicolumn{9}{|c|}{ Benign neoplasm } \\
\hline \multirow[t]{2}{*}{ Study name } & \multicolumn{5}{|c|}{ Statistics for each study } & \multicolumn{3}{|c|}{ Event rate and $95 \% \mathrm{CI}$} \\
\hline & $\begin{array}{c}\text { Event } \\
\text { rate }\end{array}$ & $\begin{array}{c}\text { Lower } \\
\text { limit }\end{array}$ & $\begin{array}{c}\text { Upper } \\
\text { limit }\end{array}$ & $Z$-Value & $p$-Value & & & \\
\hline Alghanmi et al., 2013 & 0.333 & 0.287 & 0.384 & -6.200 & 0.000 & & & \\
\hline \multirow[t]{3}{*}{ Alsham rani et al., 2019} & 0.014 & 0.009 & 0.022 & -17.522 & 0.000 & & 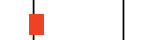 & \\
\hline & 0.212 & 0.180 & 0.247 & -12.931 & 0.000 & & & \\
\hline & & & & & -1.00 & -0.50 & 0.50 & 1.00 \\
\hline
\end{tabular}

(a)

\begin{tabular}{|c|c|c|c|c|c|c|c|c|c|}
\hline \multicolumn{10}{|c|}{ Malignant neoplasm } \\
\hline \multirow[t]{2}{*}{ Study name } & \multicolumn{5}{|c|}{ Statistics for each study } & \multicolumn{4}{|c|}{ Event rate and 95\% CI } \\
\hline & $\begin{array}{c}\text { Event } \\
\text { rate }\end{array}$ & $\begin{array}{c}\text { Lower } \\
\text { limit }\end{array}$ & $\begin{array}{c}\text { Upper } \\
\text { limit } \\
\end{array}$ & $Z$-Value & $p$-Value & & & & \\
\hline Alghanmi et al., 2013 & 0.092 & 0.066 & 0.126 & -12.557 & 0.000 & & & & \\
\hline \multirow[t]{3}{*}{ Alsham rani et al., 2019} & 0.008 & 0.004 & 0.015 & -15.166 & 0.000 & & & & \\
\hline & 0.051 & 0.038 & 0.068 & -18.447 & 0.000 & & 1 & & \\
\hline & & & & & -1.00 & -0.50 & 0.00 & 0.50 & 1.00 \\
\hline
\end{tabular}

(b)

FIGURE 3: Forest plot of the pooled proportion for the prevalence of skin cancer: (a) benign neoplasm and (b) malignant neoplasm.

hot and humid climate in both coastal cities creating good media for fungal infections. Concomitantly, infections were the most common skin disease in Cairo, Egypt (15.83\%) [24], and this finding was similarly reported in other developing countries where poor hygiene, low educational level, and poverty play important roles [25-28]. Interestingly, similar 


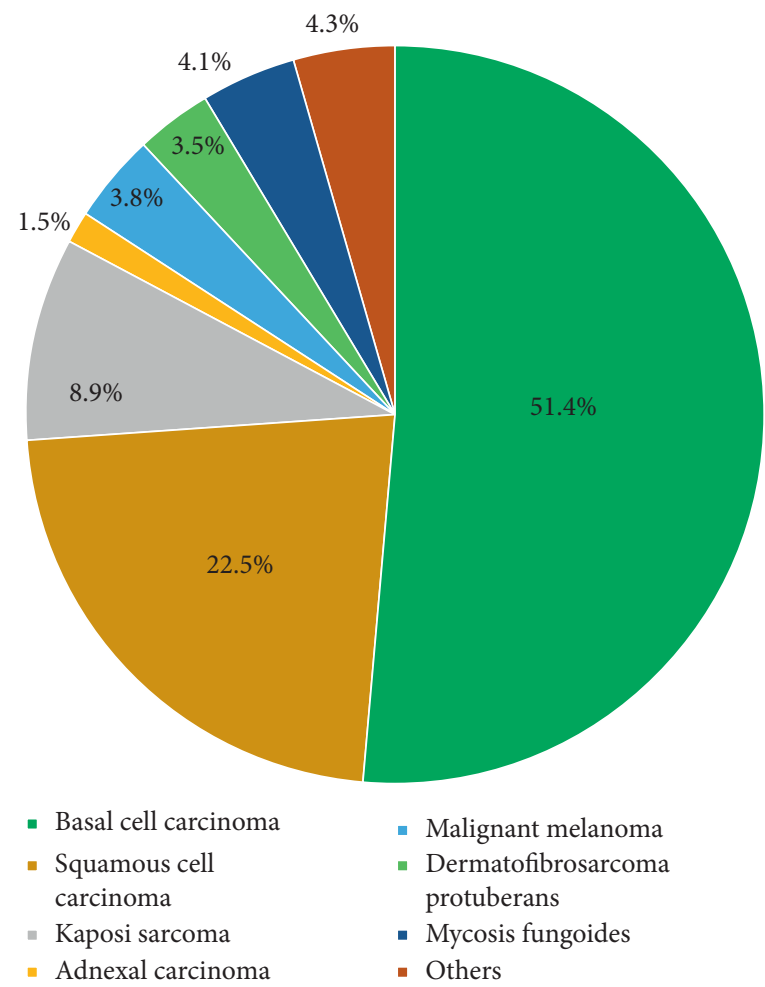

Figure 4: Pie chart summary of the pooled proportion for the prevalence malignant neoplasm.

data showed a high prevalence of fungal infection in high socioeconomic countries in the same region such as $\mathrm{Abu}$ Dhabi (8.5\%) and Qatar (11.4\%) [21]. The present study reported that the most common fungal infections were dermatophytosis and onychomycosis.

Regarding skin appendages disorders, the mean prevalence was $24.8 \%$ in Saudi Arabia. Acne served the highest proportion of them. Saudi studies reported that acne is the second most common skin disease in Jeddah, Saudi Arabia [21]. This result was also reported in Al-Khobar [8], Najran [23], Hail [12], and Al-Qunfudah [20], while acne was the third most common in Asir [15]. In Cairo, Egypt, acne represented the second most common skin disease $(6.11 \%)$, after scabies $(9.26 \%)$, contact dermatitis (7.92\%), and pityriasis versicolor (7.7\%) [24]. Diseases of skin appendages in Iran [25] and Tunisia [28] represented the second most common skin disease with significant female predominance over men among all diseases of skin appendages.

This meta-analysis reported a $5.3 \%$ prevalence of papulosquamous disorders in an overall sample size of 30076 patients from 11 studies. Psoriasis represents 3.9\%, while Lichen planus represents $1.8 \%$. Papulosquamous disorders had a higher prevalence in Jaddah (8\%), Al-jouf (7.4\%), Najran (6.7), and Al-Khobar (6.5\%). The prevalence was lower prevalence in Asir (4.8\%) and Qunfudah (4.2\%) [21].

In Cairo, Egypt, the most common skin disease was infections (45.4\%) followed by hypersensitivity diseases (22.08\%) and adnexal disorders (16.17\%). Papulosquamous disorders represented about $4.69 \%$ of the total prevalence of skin diseases. Lastly, pigmentary disorders represented only $4.24 \%$ and skin neoplasm $1.29 \%$.
In this meta-analysis of Saudi studies, the overall mean proportion of pigmentary disorders as reported by 11 studies was $16.1 \%$ from a total sample size of 16658 patients. Melanocytic nevi and vitiligo were the most common pigmentary disorders in Saudi Arabia.

Lastly, the overall prevalence of benign skin neoplasms as reported by two studies was $21.2 \%$, while malignant neoplasms were $5 \%$. Basal cell carcinoma and squamous cell carcinoma were the most common malignant cancer in Saudi Arabia. Malignant melanoma represents 3.8\% of malignant skin cancer. These figures are believed to be not representable as skin cancer is barely seen in Saudi Arabia.

The many strength points of this meta-analysis are the large numbers of included studies with a large sample size that covers most of the Saudi regions. Besides, we reported the first class of evidence form studies with low risk of bias according to the Hoy et al. [18] assessment tool for the prevalence studies.

For future researches, we recommend a comprehensive nationwide population-based study to identify the prevalence of skin diseases in Saudi Arabia.

In conclusion, Adnexal disorders and dermatitis are the most common skin disease in Saudi Arabia followed by skin infection and pigmentary disorders. Dermatological educational programs for primary healthcare physicians should be implemented to provide rapid detection of cases at early stagesbib19.

\section{Conflicts of Interest}

The authors declare that they have no conflicts of interest. 


\section{Acknowledgments}

The author would like to thank Dr. Malik Almohideb (2nd reviewer) (email: malikalm@hotmail.com, Affiliation: Internal Medicine, Prince Sultan Military Medical City, Riyadh, Saudi Arabia) and Dr. Hussein Ahmed (3rd reviewer) (email: hoseen011232@medicine.zu.edu.eg, Affiliation: Faculty of Medicine, Zagazig University, Zagazig, Egypt) for their help while conducting this meta-analysis.

\section{Supplementary Materials}

Supplementary file 1: risk of bias assessment tool. Supplementary file 2: forest plot of the pooled proportion for the prevalence of pigmentary disorders. Supplementary file 3: forest plot of the pooled proportion for the prevalence of dermatitis/eczema and related conditions. Supplementary file 4: forest plot of the pooled proportion for the prevalence of skin infections. Supplementary file 5: forest plot of the pooled proportion for the prevalence of diseases of skin appendages. Supplementary file 6: forest plot of the pooled proportion for the prevalence of papulosquamous disorders. (Supplementary Materials)

\section{References}

[1] C. Korfitis, S. Gregoriou, C. Antoniou, A. D. Katsambas, and D. Rigopoulos, "Skin biopsy in the context of dermatological diagnosis: a retrospective cohort study," Dermatology Research and Practice, vol. 2014, Article ID 734906, 5 pages, 2014.

[2] L. K. Andersen and M. D. P. Davis, "The epidemiology of skin and skin-related diseases: a review of population-based studies performed by using the Rochester epidemiology project," Mayo Clinic Proceedings, vol. 88, no. 12, pp. 1462-1467, 2013.

[3] L. P. Fox, J. Cotliar, L. Hughey, D. Kroshinsky, and K. Shinkai, "Hospitalist dermatology," Journal of the American Academy of Dermatology, vol. 61, no. 1, pp. 153-154, 2009.

[4] A. A. Alasmari, A. H. Hakeem, F. S. Bin Saleh et al., "Pattern of dermatological disease encountered in a hematology ward: a retrospective analysis of dermatology consultation in a hematology ward in a tertiary care center in Saudi Arabia," Dermatology Research and Practice, vol. 2019, Article ID 9891270, 5 pages, 2019.

[5] V. Alsaigh, L. A. Schachner, V. Rae et al., "Dermatologic consultations in the hospital setting," Archives of Dermatology, vol. 130, no. 8, pp. 1022-1025, 1994.

[6] A. Albasri and W. Borhan, "Histopathological pattern of skin cancer in western region of Saudi Arabia. An 11 years experience," Saudi Medical Journal, vol. 39, no. 10, pp. 994-998, 2018.

[7] A. Albasri and I. Ansari, "The histopathological pattern of benign and non-neoplastic skin diseases at King Fahad Hospital, Madinah, Saudi Arabia," Saudi Medical Journal, vol. 40, no. 6, pp. 548-554, 2019.

[8] O. M. Alakloby, "Pattern of skin diseases in Eastern Saudi Arabia," Saudi Medical Journal, vol. 26, no. 10, pp. 1607-1610, 2005.

[9] N. M. Alghanmi and L. S. Abdullah, "Pathology of skin diseases. A study from Western Saudi Arabia," Saudi Medical Journal, vol. 34, no. 1, pp. 74-79, 2013.
[10] J. A. Al-Maghrabi, A. S. Al-Ghamdi, and H. A. Elhakeem, "Pattern of skin cancer in Southwestern Saudi Arabia," Saudi Medical Journal, vol. 25, no. 6, pp. 776-779, 2004.

[11] W. Y. Al-Saeed, K. M. Al-Dawood, I. A. Bukhari, and A. A. Bahnassy, "Prevalence and pattern of skin disorders among female schoolchildren in Eastern Saudi Arabia," Saudi Medical Journal, vol. 27, no. 2, pp. 227-234, 2006.

[12] F. Al Shammrie and A. Al Shammrie, "Pattern of skin disease in Hail region of Saudi Arabia," Journal of Dermatology \& Dermatologic Surgery, vol. 21, no. 2, pp. 62-65, 2017.

[13] F. T. Alshammari, S. M. Alghaythi, and M. A. Alharbi, "Profile of skin biopsies and skin cancer subtypes in Hail Region: a three years retrospective study," The Egyptian Journal of Hospital Medicine, vol. 70, no. 1, pp. 177-180, 2018.

[14] H. A. Al Shobaili, "The pattern of skin diseases in the Qassim region of Saudi Arabia: what the primary care physician should know," Annals of Saudi Medicine, vol. 30, no. 6, pp. $448-453,2010$.

[15] K. A. Bahamdan, J. U. Egere, A. K. Khare, T. Tallab, K. Ibrahim, and M. M. Mourad, "The pattern of skin diseases in Asir region, Saudi Arabia: a 12-month prospective study in a referral hospital," Annals of Saudi Medicine, vol. 15, no. 5, pp. 455-457, 1995.

[16] J. P. Higgins and S. Green, Cochrane Handbook for Systematic Reviews of interventions, The Cochrane Collaboration, London, U K, 2008.

[17] D. Moher, A. Liberati, J. Tetzlaff, and D. G. Altman, "Preferred reporting items for systematic reviews and meta-analyses: the PRISMA statement," PLoS Medicine, vol. 6, no. 7, Article ID e1000097, 2009.

[18] D. Hoy, P. Brooks, A. Woolf et al., "Assessing risk of bias in prevalence studies: modification of an existing tool and evidence of interrater agreement," Journal of Clinical Epidemiology, vol. 65, no. 9, pp. 934-939, 2012.

[19] J. P. Blyth and S. Green, "Cochrane handbook for systematic reviews of intervention," Cochrane Book Series, pp. 1-649, London, UK, 2008.

[20] A. Ahmed, A.-A. Ali, A. Hussain, and A. Hussain, "The pattern of skin diseases in a hospital in Qunfudah region, Saudi Arabia," International Journal of Advanced Research, vol. 4, no. 12, pp. 1365-1370, 2016.

[21] H. M. Hussain, M. A. Alsolami, A. M. Alshehri et al., "Pattern of skin diseases in a university hospital in Jeddah, Saudi Arabia: age and sex distribution," Annals of Saudi Medicine, vol. 39, no. 1, pp. 22-28, 2019.

[22] A. Salman and A. F. Al Gufai, "The pattern of skin diseases in Hail region, Saudi Arabia," Annals of Saudi Medicine, vol. 18, no. 6 , pp. $558-561,1998$.

[23] H. H. Shelleh and H. S. Al-Hatiti, "Pattern of skin diseases in a hospital in Southwestern Saudi Arabia," Saudi Medical Journal, vol. 25, no. 4, pp. 507-510, 2004.

[24] E. A. El-Khateeb, A. A. Imam, and M. A. Sallam, "Pattern of skin diseases in Cairo, Egypt," International Journal of Dermatology, vol. 50, no. 7, pp. 844-853, 2011.

[25] S. Baghestani, S. Zare, and A.-A. Mahboobi, "Skin disease patterns in Hormozgan, Iran," International Journal of Dermatology, vol. 44, no. 8, pp. 641-645, 2005.

[26] M. Lal Khatri, "Spectrum of skin diseases in Yemen (Hajjah and adjacent region)," International Journal of Dermatology, vol. 43 , no. 8 , pp. $580-585,2004$.

[27] A. O. Ogunbiyi, O. O. M. Daramola, and O. O. Alese, "Prevalence of skin diseases in Ibadan, Nigeria," International Journal of Dermatology, vol. 43, no. 1, pp. 31-36, 2004. 
[28] A. Souissi, F. Zeglaoui, B. Zouari, and M. R. Kamoun, "A study of skin diseases in Tunis. An analysis of 28,244 dermatological outpatient cases," Acta Dermatovenerologica Alpina, Pannonica, Adriat, vol. 16, no. 3, pp. 111-116, 2007. 